\title{
Análisis comparativo de las leyes para el ejercicio de la Enfermería en Paraguay, El Salvador, Argentina y Perú
}

\author{
Musayón-Oblitas Yesenia ${ }^{1}$, Farro-Peña Gianina ${ }^{1}$, Torres-Deza Clara ${ }^{1}$, Alayo-Sarmiento Margarita ${ }^{1}$, \\ Loncharich-Vera Natalie ${ }^{1}$, Zambonini Silvia ${ }^{1}$, Felippa Gabriela ${ }^{1}$, Spadafora Santiago ${ }^{1}$, Osa María ${ }^{1}$, \\ Robles Néstor ${ }^{1}$, Rolón Violeta ${ }^{1}$, Figueroa-Gallardo Tomas ${ }^{1}$, Godines-Valencia Carlos ${ }^{1}$
}

\section{RESUMEN}

Las legislaciones ayudan a civilizaciones democráticas a alcanzar orden en su actuar y dar legalidad a sus procedimientos. Las legislaciones brindadas para el ejercicio de enfermería permiten al profesional enfermero ejercer sus funciones con respaldo legal, delimitando su actuar y sancionando a quien vulnere el orden en su proceder. El presente artículo tiene por objetivo analizar la legislación vigente para el ejercicio de la enfermería en Paraguay, El Salvador, Argentina y Perú, para lo cual se presenta y revisa los principales dictámenes de las legislaciones en cada uno de los países, contrastando sus principales aportes para la práctica de enfermería desde la formación de la carrera hasta su ejercicio en la sociedad.

Palabras clave: a. Perú. (Fuente DeCs BIREME).

\section{Comparative analysis of the laws for the exercise of nursing in Paraguay, El Salvador, Argentina and Peru}

\begin{abstract}
Laws help achieve democratic civilizations in order to give legitimacy to act and its procedures. Legislation offered to nursing practice enable the professional nurse perform his duties with legal support, limiting their actions and punishing who violates the order in its action. This article aims to analyze the current legislation for nursing practice in Paraguay, El Salvador, Argentina and Peru, for which introduces and reviews the main opinions of the laws in each country, contrasting its main contributions for nursing practice since the formation of the race until his tenure at the company.
\end{abstract}

Key words: A. Perú. (Fuente DeCs BIREME).

Desde el año 1977, la Organización Internacional de Trabajo (OIT) declaró que: El personal de enfermería comprende todas las categorías de personal que presta asistencia y servicios de enfermería (1); esto incluye al personal profesional y al personal técnico y auxiliar, cuya formación se brinda en instituciones de educación superior no universitaria y, en algunos lugares, en instituciones donde se brinda educación para el trabajo. La OIT también señala que los estados suscritos a ese convenio internacional deberán tomar medidas para proporcionar Educación y formación apropiada para el ejercicio de las funcio- nes del personal de enfermería, así como asegurar Condiciones de empleo y trabajo..., capaces de atraer y retener al personal (1).

A lo largo de los años, enfermeras de la región, apoyadas en ocasiones por los gobiernos de turno, han emprendido innumerables esfuerzos por hacer de la enfermería una práctica reglamentada. Sin embargo, el crecimiento no ha sido homogéneo en toda América Latina, LO cual ha ocasionado disparidades en su reglamentación.

Integrantes del Proyecto ALFA. Funda Enfermerie. 


\section{De los conceptos y alcances:}

En Argentina se reconocen, de acuerdo con la Ley 24.004/ 91 «Régimen laboral del ejercicio de la Enfermería», en el ámbito sometido a la jurisdicción Nacional, dos niveles para el ejercicio de esta profesión: enfermero/a profesional y enfermero/a auxiliar (2).

Por otro lado, en el Art. 5..$^{\circ}$ de la Ley 298/99 de la Ciudad Autónoma de Buenos Aires se reconocen también dos niveles, incorporando en el primer nivel a los licenciados/ as en Enfermería.

El primer nivel tiene autonomía y aplica un cuerpo sistemático de conocimientos para la identificación y resolución de las situaciones de salud - enfermedad sometida al ámbito de su competencia. El segundo nivel consiste en la práctica de técnicas y conocimientos que contribuyen al cuidado de enfermería, planificado y dispuesto por el nivel profesional y ejecutado bajo su supervisión. Este orden jurídico está dictado desde el año 1991 a nivel nacional, y sigue vigente en la actualidad. En Argentina, el ejercicio de la Enfermería profesional está reservado solo para aquellas personas que posean el título habilitante otorgado por universidades nacionales, provinciales o privadas, centros de formación terciarios (no universitarios) dependientes de organismos nacionales, provinciales o municipales o instituciones privadas, en ambos casos reconocidas por la autoridad competente. También son reconocidos como profesionales enfermeros quienes presenten un diploma extranjero que los acredite como tal, previa revalidación.

Por su parte, se reconoce como personal auxiliar de Enfermería a aquellos que tengan un certificado correspondiente otorgado por instituciones nacionales, provinciales o municipales, o instituciones privadas, reconocidas por la autoridad competente.

Sin embargo, a pesar de realizar esta clara delimitación entre el título profesional y auxiliar, en la Ley 24.004, del régimen legal del ejercicio de Enfermería; establece en sus artículos 9, 10 y 11 los mismos derechos, obligaciones y prohibiciones tanto para el personal profesional como para el auxiliar de Enfermería.

Para la legislación Argentina, el ejercicio de la Enfermería comprende las funciones de: Promoción, recuperación y rehabilitación de la salud, así como la de prevención de enfermedades, realizadas en forma autónoma dentro de los límites de competencia que deriva de las incumbencias de los respectivos títulos habilitantes.
Así mismo, será considerado ejercicio de la Enfermería la docencia, la investigación y el asesoramiento sobre temas de su incumbencia, y administración de servicios, cuando sean realizados por las personas autorizadas por la presente, a ejercer la Enfermería.

En Argentina, un enfermero, técnico o auxiliar, tiene derecho a ejercer su profesión de conformidad con la ley, asumir la responsabilidad que le deriva de tal ejercicio y negarse a realizar prácticas que contravengan con sus convicciones religiosas, morales o éticas, siempre que de ello no resulte un daño inmediato o mediato en el paciente sometido a esa práctica. Este desenvolvimiento profesional lo realizará en ambientes laborales con las debidas garantías.

El profesional, técnico o auxiliar de Enfermería está obligado a respetar los derechos de las personas, entre ellos muy especialmente el derecho a la vida, la salud y la dignidad. Debe colaborar con la autoridad sanitaria cuando se requiera, mantener la idoneidad de su labor y respetar el secreto profesional.

Les está prohibido realizar prácticas que vayan en menoscabo de la salud y bienestar de las personas, no pueden ejercer su profesión mientras tenga una enfermedad infectocontagiosa o cualquier otra enfermedad inhabilitante, situación que deberá ser fehacientemente comprobada por la autoridad sanitaria, y publicar información que induzca al engaño público.

Según el artículo 12 de la Ley 24.004 para el ejercicio de la Enfermería, tanto en el nivel profesional como en el auxiliar, se debe inscribir previamente el título, diploma o certificado habilitante en la Subsecretaría de Salud del Ministerio de Salud y Acción Social, la que autorizará el ejercicio de la respectiva actividad, otorgando la matrícula y extendiendo la correspondiente credencial (2). Es esta misma institución quien podrá ejercer labor disciplinaria cuando el caso lo requiera.

Un registro o matriculación puede quedar sin efecto cuando el interesado lo solicite, por sanción o por fallecimiento. La sanción disciplinaria puede llevarse a cabo cuando exista condena judicial, no se cumpla la ley del ejercicio de la Enfermería o exista negligencia frecuente, ineptitud u omisión grave.

En Perú, por su parte, la legislación vigente data recién del año 2002, así como su reglamentación, y tienen un ámbito de aplicación nacional, en ella se incluye al profe- 
sional de enfermería de sectores laborales públicos y de privados. La Ley precisa también que ante cualquier circunstancia de la señalada previamente se buscará el «beneficio del enfermero»; quien además lo reconoce cuando es colegiado. El registro y la colegiación respectiva es otorgada por el Colegio de Enfermeros del Perú, creado en el año 1978 (3).

A diferencia de la legislación Argentina, la Ley del Enfermero Peruano, es exclusiva para el profesional licenciado en enfermería, no incluye al personal técnico. Para realizar el ejercicio de la profesión se requiere contar con el título universitario a nombre de la Nación, y prohíbe la denominación de enfermero o enfermera a quien carezca de este título, bajo aplicación del artículo 363 del Código Penal, el cual establece que: Quien ejerce profesión sin reunir los requisitos legales requeridos o presentando un título falso, será reprimido con pena privativa de libertad (4).

Son derechos de un enfermero en el Perú: el acceder a cargos de dirección o gerencia en igualdad de condiciones que otros profesionales de salud; percibir una remuneración equitativa y justa según escalafón; contar con un ambiente laboral seguro, y disponer de materiales necesarios para ejercer su labor; disfrutar de licencia con goce de haber para determinados cargos de representación nacional o internacional, someterse a exámenes médicos preventivos, y ser contratados bajo la modalidad y plazos que corresponda a la naturaleza de sus funciones. En correspondencia, es obligación del enfermero cumplir con el código de ética y deontología de la profesión y conocer y aplicar la legislación vigente.

En la legislación peruana no se menciona niveles de formación, pues reconoce que el profesional de enfermería es solo quien egresa de una universidad y recibe el título profesional respectivo, se menciona más bien, niveles de capacitación y perfeccionamiento para aquellos enfermeros que se especializan en un área de cuidado, los cuales deben ser aprobados por el Colegio de Enfermeros del Perú.

El profesional de Enfermería peruano puede desenvolverse en las áreas de docencia, administración, asistencial e investigación, y tiene como función brindar el cuidado integral de enfermería basado en el proceso de atención de enfermería, encomendar actividades de menor complejidad al personal no profesional de enfermería bajo su supervisión y responsabilidad, conducir los servicios, gestionar programas de formación, entre otros.
En El Salvador, el profesional enfermero no cuenta con una norma jurídica que delimite su quehacer. Es la Constitución de la República del Salvador quien a partir del reconocimiento de la salud de los habitantes como un «bien público» establece, en el artículo 67, carreras sanitarias, hospitalarias, paramédicas y de administración hospitalaria, y en el artículo 68 dictamina que el Consejo Superior de Salud Pública «velará por la salud del pueblo» y estará conformado por los gremios médico, odontológico, químico-farmacéutico y médico veterinario (5). En la Constitución no se menciona a la Enfermería, ni como carrera ni como gremio.

En el año 1992 el Decreto 2699 estableció la «Ley del Consejo Superior de Salud Pública y de las Juntas de Vigilancia de las Profesiones de Salud», donde se reconoce a la profesión de enfermería y se establece que será «objeto de vigilancia por un organismo legal» denominado «Junta de Vigilancia de la Profesión de Enfermería». Literalmente, el mismo artículo menciona que: Se entenderá que forman parte del ejercicio de las profesiones antes mencionadas, y por consiguiente estarán sometidas a la respectiva Junta de Vigilancia, aquellas actividades especializadas, técnicas y auxiliares que sean de complemento de dichas profesiones. Lo que implica que quedan reconocidas las labores técnicas de enfermería (6). La reglamentación existente tiene ámbito de aplicación nacional.

En el Salvador, por Decreto 468, se pone en vigencia la Ley de Educación Superior, y en ella se identifican la educación tecnológica y la educación universitaria como parte de la educación superior (7).

Después de su formación, tanto profesionales como técnicos, ejercen las funciones que el estado determina a través del «Código de Salud». En El Salvador, el «Código de Salud», establecido por decreto 955, desarrolla los principios constitucionales relacionados con la salud pública de la población salvadoreña, y determina que las obligaciones del personal de salud, incluido los profesionales y técnicos de enfermería, son: Atender a las personas que así lo requieran incluyendo los casos de emergencia, cumpliendo la reglamentación vigente incluyendo la de índole ético. Las precisiones de las normas éticas para el profesional de Enfermería son determinadas por la Junta de Vigilancia a través del «Código de Ética para Profesionales de Enfermería».

El «Código de Salud» establece también como derechos de los profesionales, técnicos e higienistas, el poder ser contratados, velar por la superación de su gremio, ser 
amparado por su junta respectiva y transferir a los pacientes cuando así lo consideren conveniente. Finalmente, el mismo código prohíbe engañar con falso alivio, permitir el uso de su nombre a otro ante la junta, difamar, calumniar, entre otros.

El ejercicio de cada profesión comprende la prescripción, elaboración, administración, indicación o aplicación de cualquier procedimiento directo o indirecto destinado al diagnóstico, pronóstico y tratamiento de las enfermedades, con el propósito de realizar acciones de prevención, promoción, protección y recuperación de la salud de las personas, así como también de asesoramiento público, privado y pericial... así lo establece el «Código de Salud». Pero solo hace precisiones para las actividades técnicas y auxiliares de la profesión médica, odontológica y químicafarmacéutica (8). En el artículo 35 prohíbe expresamente a los técnicos y auxiliares, solo en el rubro dental, la atención clínica o quirúrgica de los pacientes. El ejercicio de una profesión está regulada por la Junta respectiva.

La Ley 3206 del Ejercicio de la Enfermería, publicada el 13 de junio de 2007 (9) y su reglamento aprobado el 6 de diciembre del mismo año (10), se aplica en la republica de Paraguay. Según el ejercicio, la profesión esta en relación con: El cuidado de la salud del individuo, familia y comunidad, tomando en cuenta la promoción de la salud y calidad de vida, la prevención de la enfermedad y la participación de su tratamiento, incluyendo la rehabilitación de la persona, independientemente de la etapa de crecimiento y desarrollo en que se encuentre, debiendo mantener al máximo, el bienestar físico, mental, social y espiritual del ser humano. Adicionalmente a ello, la enfermera/o puede desempeñarse en la docencia e investigación, basándose en los principios científicos, conocimientos y habilidades adquiridas en su formación profesional, actualizándose mediante la experiencia y educación continua.

El ámbito de aplicación de la Ley comprende el ejercicio profesional, las áreas de docencia, administración e investigación en todas las dependencias públicas y privadas a nivel nacional. Sin embargo, no hay una clara delimitación entre la formación profesional y técnica, puesto que se dictamina que para el ejercicio de la «profesión de Enfermería» se requiere haber realizado estudios superiores o técnicos, pudiendo concluir como auxiliar, técnico o profesional.

En adelante, la legislación Paraguaya incluye en esta categoría a todo el personal de enfermería, no haciendo una clara precisión entre el rol que cumple uno de otro.
Para realizar el ejercicio «de la profesión de Enfermería», la Ley precisa que se requiere del título de licenciado, técnico o auxiliar, los que deben estar inscritos en el Ministerio de Salud Pública y Bienestar Social.

En el caso de que surja alguna situación que requiera acción disciplinaria, es el Ministerio de Salud Pública y Bienestar Social quien ejercerá el poder disciplinario. Las sanciones pueden ir desde una llamada de atención hasta la suspensión o cancelación de su matrícula.

En cuanto al régimen laboral de Paraguay se señala una jornada laboral del personal de enfermería con un máximo de treinta horas semanales.

El descanso remunerado correspondiente a los días feriados no laborales será contabilizado dentro de la jornada semanal; asimismo, se reconoce las horas extras que deben ser remuneradas según lo establecido.

Análisis comparativo de las leyes para la formación de enfermeros en Paraguay, El Salvador, Argentina y Perú

La educación es una de las más grandes responsabilidades que tiene la sociedad para combatir la pobreza, el subdesarrollo la exclusión, y asegurar la seguridad, la justicia, la libertad y la paz de los pueblos (11). Para la UNESCO: Nunca antes en la historia el bienestar de las naciones ha estado tan estrechamente vinculado a la calidad y el alcance de sus sistemas e instituciones de enseñanza superior (12).

A lo largo de los años los países han dictaminado una serie de políticas públicas destinadas a asegurar el acceso a la educación, desde la educación básica hasta le educación superior. Muchas de estas políticas están plasmadas en las leyes de educación, las que no son homogéneas en América Latina; a continuación se hace un análisis de sus principales similitudes y diferencias.

En Argentina, de acuerdo con lo establecido por la Ley de Educación Nacional 26.206/06, la estructura del sistema educativo comprende cuatro (4) niveles (educación inicial, educación primaria, educación secundaria y educación superior), y ocho (8) modalidades; el artículo 15 determina la posibilidad de «articulación» entre estas modalidades. El estado garantiza la inclusión, acceso y gratuidad de la enseñanza en todos los niveles de formación y en sus diferentes modalidades, y establece la obligatoriedad desde la edad de 5 años hasta la finalización de la escuela secundaria (13). 
La educación superior está regulada por la Ley de Educación Superior 24.521/95 (14) que establece una estructura del sistema constituida por instituciones de educación superior no universitaria (reguladas por la Ley de Educación Técnico Profesional 26.058) (15), que abarca la formación docente, humanística, social, técnico-profesional o artística; y por instituciones de educación universitaria, que comprende universidades e institutos universitarios. Para ingresar como alumno a las instituciones de nivel superior se debe haber aprobado el nivel medio o el ciclo polimodal de enseñanza. Excepcionalmente, los mayores de 25 años que no reúnan esa condición, podrán ingresar siempre que demuestren, a través de las evaluaciones que las provincias, la Municipalidad de la Ciudad de Buenos Aires o las universidades en su caso establezcan; que tienen preparación o experiencia laboral acorde con los estudios que se proponen iniciar, así como aptitudes y conocimientos suficientes para cursarlos satisfactoriamente.

Esta Ley posibilita la «articulación»entre las distintas instituciones que conforman el Sistema de Educación Superior, que tienen por finalidad facilitar el cambio de modalidad; de orientación o carrera; la continuación de los estudios en otros establecimientos sean universitarios o no, así como la reconversión de los estudios concluidos.

Corresponde exclusivamente a las instituciones universitarias otorgar el título de grado de licenciado y títulos profesionales equivalentes, así como los títulos de posgrado de especialista, magister y doctor. El reconocimiento oficial de los títulos que expidan las instituciones universitarias será otorgado por el Ministerio de Cultura y Educación. Los títulos oficialmente reconocidos tendrán validez nacional, certificarán la formación académica recibida y habilitarán para el ejercicio profesional respectivo en todo el territorio nacional.

La formación del personal de Enfermería se da en el nivel superior, tanto en el ámbito universitario, como en el ámbito no universitario. En el ámbito universitario se otorga el título de licenciado en Enfermería para quienes estudian 4 o 5 años, o enfermero para quienes cursan un nivel de formación intermedio de 3 años.

Mientras que en el ámbito no universitario quienes estudian en centros de formación terciaria en Enfermería reciben el título de enfermero y estudian 3 años; mientras que el título de auxiliar lo reciben quienes estudian 9 meses.

Por último, la Ley de Educación Superior prevé en el artí- culo 43 que en el caso de títulos correspondientes a profesiones reguladas por el Estado, cuyo ejercicio pudiera comprometer el interés público poniendo en riesgo de modo directo la salud, la seguridad, los derechos, los bienes o la formación de los habitantes, deberán ser acreditadas periódicamente por la Comisión Nacional de Evaluación y Acreditación Universitaria (CONEAU) o por entidades privadas constituidas con ese fin debidamente reconocidas.

En el caso de Enfermería, la carrera aún no ha sido incorporada al artículo mencionado, por lo tanto, no ha habido convocatoria de acreditación a nivel nacional. No obstante, algunas carreras que se dictan en universidades nacionales han participado de un proceso regional de acreditación de carreras de Enfermería, correspondiente al Sistema ARCU-SUR (Sistema de Acreditación de Carreras Universitarias para el Reconocimiento Regional de la Calidad Académica de sus respectivas Titulaciones en el MERCOSUR y estados asociados).

En el Perú, la educación es gratuita y obligatoria según su Constitución Política (16). El sistema educativo peruano se encuentra conformado por la educación básica regular, la que se brinda en tres niveles: inicial, primaria y secundaria. En el caso de la universidad, la educación es gratuita en las universidades públicas para aquellos estudiantes que mantengan un buen rendimiento académico.

La educación inicial se inicia a los 3 años, y su objetivo es favorecer el desarrollo integral del niño, quien a partir de los 5 y 6 años debe empezar la educación primaria hasta su término en el sexto grado. Este nivel se agrupa en tres ciclos, en el primer ciclo se ubica el primer y segundo grado, en el segundo ciclo el tercer y cuarto grado y el en el tercer ciclo se ofrece el quinto y sexto grado. A partir de allí, el estudiante pasa al nivel secundario. El nivel secundario se ofrece en cinco años de formación.

Además de la educación básica regular, tenemos: la educación básica especial y la educación básica alternativa (la primera se ofrece a aquellos con alguna discapacidad y la segunda a los estudiantes que por alguna razón no tuvieron acceso a la educación básica regular), también se incluye a la educación intercultural bilingüe y rural que busca la inclusión de pueblos indígenas y la diversidad cultural.

La educación superior se ofrece en tres años, y tiene énfasis tecnológico y técnico productivo. Pueden estudiar en los institutos todos aquellos jóvenes que hayan conclui- 
do los estudios secundarios. Esta educación lo brindan los institutos y ofrecen el título de «profesional técnico», cuando el estudiante haya concluido y aprobado todos los cursos $\mathrm{o}$ asignaturas.

Finalmente, se tiene la educación universitaria, que se ofrece en las universidades. Pueden acceder a los estudios universitarios todos los jóvenes que hayan terminado los estudios secundarios y que concluyan con éxito el proceso de admisión universitario, o aprueben un examen. Solo las instituciones universitarias pueden otorgar a nombre de la Nación el grado de bachiller y el título universitario. El grado se obtiene automáticamente al aprobar los años de formación requeridos que no son menos de cinco o de diez semestres académicos. El título universitario se obtiene con el examen de grado o con la sustentación de un trabajo científico o tesis (17).

Los estudios en todos los niveles reconocidos por el estado peruano no son articulados. Algunas universidades reconocen algunos cursos de estudiantes provenientes de institutos, acorde a su curricular de formación, pero no es una norma ni reglamentación.

El Sistema Nacional de Calidad, Evaluación y Certificación (SINEACE), por Ley 28740, debe promover la calidad educativa en el país desde el nivel básico, tecnológico y universitario (18). El SINEACE cuenta con tres operadores: para le educación básica se encuentra el Instituto Peruano de Evaluación, Acreditación y Certificación de la Calidad de la Educación Básica (IPEBA). El Consejo de Evaluación, Acreditación y Certificación de la Calidad de la Educación Superior no Universitaria (CONEACES), es el segundo operador, encargado de velar por la excelencia en los procesos, servicios y resultados educativos de la educación superior no universitaria; mientras que el Consejo de Evaluación, Acreditación y Certificación de la Calidad de la Educación Superior Universitaria (CONEAU), vela por la calidad del educación universitaria, siendo el tercer operador (18).

La formación de personal de Enfermería se da solo en dos niveles. Los institutos tecnológicos ofrecen la carrera de enfermería técnica, y otorgan el título de profesional técnico en Enfermería. Los estudiantes estudian tres años, y su ámbito de competencia es básico, brindan atención a los pacientes bajo la supervisión y responsabilidad del licenciado en Enfermería.

Para obtener el título de licenciado en Enfermería se debe postular a una vacante en la universidad y después del ingreso aprobar los cinco años de formación o diez semestres académicos. El grado de bachiller en enfermería se obtiene automáticamente, y el título de licenciado después de aprobar el examen de grado o sustentar una tesis. Para ejercer la carrera todo licenciado en enfermería debe obtener su registro o colegiarse en el Colegio de Enfermeros del Perú.

En el Perú no existe la profesionalización de técnicos, y no existe tampoco articulación entre los estudios técnicos y profesionales.

En El Salvador según la Ley General de Educación de 1990 (19), La educación parvularia y la básica es obligatoria y juntamente con la especial será gratuita cuando la imparta el estado. El sistema educativo se divide en dos modalidades formal e informal.

Según el artículo 9 de la misma Ley: La Educación Formal es la que se imparte en establecimientos educativos autorizados, en una secuencia regular de años o ciclos lectivos, con sujeción a pautas, curriculares progresivas y conducentes a grados y títulos. Sus niveles son: inicial, parvulario, básico, medio y superior.

La educación no formal se brinda con el objetivo de actualizar, completar o suplir conocimientos, y responde a las necesidades de las personas y la sociedad.

El nivel inicial comienza con el nacimiento del niño y se prolonga hasta los cuatro años de edad, no es obligatoria, ni gratuita. Favorecerá el desarrollo socio-afectivo, psicomotriz, censo-perceptivo, de lenguaje del niño y se centrará en la familia y la comunidad.

La educación parvularia se ofrece a niños de 4 a 6 años de edad, es gratuita pero no es obligatoria. Tiene por objetivo el desarrollo psicomotor y de lenguaje, de los niños agrupados según a su edad. La educación básica, en El Salvador, es gratuita y obligatoria y atiende a niños desde los 7 años de edad, se agrupa en tres ciclos de tres años cada uno. La educación media (bachillerato diversificado), se brinda en dos modalidades: una general y otra técnicovocacional, ambas permitirán continuar con estudios superiores o incorporarse a la actividad laboral. Este nivel no es obligatorio, ni es gratuito, y es necesario aprobar un examen de admisión.

La educación superior se imparte en los institutos tecnológicos y en las escuelas de educación superior. Los institutos ofrecen carreras en dos o tres años y sus gradua- 
dos pueden acceder a la universidad. Por su parte, las escuelas ofrecen carreras de tres y cuatro años. Los programas académicos de ambas instituciones son similares, pero la escuela superior enfatiza en la formación básica y personal. Según el artículo 4 de la Ley de Educación Superior: La educación tecnológica, tiene como propósito la formación y capacitación de profesionales y técnicos especializados en la aplicación de los conocimientos y destrezas de las distintas áreas científicas o humanísticas.

Para seguir estudios universitarios los jóvenes deben aprobar un examen de admisión y completar un mínimo de cuatro años de estudio. Por ley, La educación universitaria es aquella que se orienta a la formación en carreras con estudios de carácter multidisciplinario en la ciencia, el arte, la cultura y la tecnología, que capacita científica y humanísticamente, y conduce a la obtención de los grados universitarios.

La educación superior otorga, en El Salvador, los grados de técnico, profesor, tecnólogo, licenciado, ingeniero y arquitecto, maestro, doctor y especialista.

La Ley de Educación, también incluye la educación de adultos, la educación especial, y la educación artística.

El Ministerio de Educación es quien establece los planes de formación y es el encargado de velar por la calidad. Las instituciones son las responsables de realizar los registros respectivos y solo el Ministerio de Educación puede otorgar equivalencias de estudio a toda persona que los haya realizado en el extranjero y desee incorporarse al sistema educativo de El Salvador.

En el caso de los estudios en Enfermería, esta se ofrece en tres niveles educativos: técnico (dos años), tecnólogo (tres años) y licenciatura (cinco años).

La licenciatura es ofrecida por las universidades facultadas de desarrollar los tres niveles, quien otorga el título de licenciado en Enfermería.

El título de técnico en enfermería es otorgado por un instituto superior, donde se estudia de 2 a 3 años, y está facultado para formar en solo dos niveles (técnico y tecnólogos). Los auxiliares, ya no se forman en El Salvador por disposición legal. La Ley vigente no permite articulación entre los distintos niveles de formación del personal de enfermería.

En Paraguay los estudios de Enfermería se ofrecen en dos niveles educativos. La licenciatura es ofrecida en la universidad quien otorga el título de licenciado en Enfermería.
La certificación del técnico en Enfermería es otorgado por un instituto superior, donde se estudia de 2 a 3 años. Los auxiliares estudian en un centro o instituto educativo por un año. Los grados y posgrados son otorgados por la universidad. La ley vigente no permite articulación entre los distintos niveles de formación del personal de enfermería, por lo cual no se validan los años de estudios de un auxiliar o técnico para la obtención de la licenciatura.

Actualmente,la Agencia Nacional de Evaluación y Acreditación de la Educación Superior (ANEAES) bajo la dirección del Ministerio de Educación y Cultura de Paraguay, pero con autonomía técnicay académica para el cumplimiento de sus funciones, viene realizando diversas acciones para realizar la acreditación de diferentes carreras a nivel nacional, entre ellas Enfermería (20).

Las universidades pueden otorgar los siguientes títulos: técnico, de grado académico y de posgrado. Los títulos de posgrado abarcan especialización, maestría y doctorado.

Los institutos superiores pueden otorgar títulos de grado que habilitan a la profesión y, de acuerdo con el Art. 54 de la Ley General de Educación, son también responsables de la educación de posgrado, por lo mismo, pueden otorgar títulos de posgrado (21).

Los institutos técnicos superiores otorgan títulos de su nivel, asimismo, los institutos de formación docente otorgan títulos correspondientes a profesores (Educación Inicial, Educación Escolar Básica y Educación Media).

Actualmente, se encuentra en estudio en el Poder Legislativo un anteproyecto de Ley de Educación Superior. Normativa muy necesaria para actualizar los fines y dinamizar la estructura organizacional y académica de este nivel.

Para subsanar el vacío legislativo en el ámbito de educación superior, por Decreto de la Presidencia de la República se conformó una Comisión ad hoc encargada de la reforma de la educación superior que tiene entre sus funciones elaborar en consenso un anteproyecto de Ley de Educación Superior que reemplace a la Ley 136 de Universidades (22).

«La presente publicación ha sido elaborada con la asistencia de la Unión Europea, en el marco del proyecto Alfa Funda Enfermería (Contrato CRIS 279-078). El contenido de la misma es responsabilidad exclusiva de los autores y en ningún caso debe considerarse que refleja los 


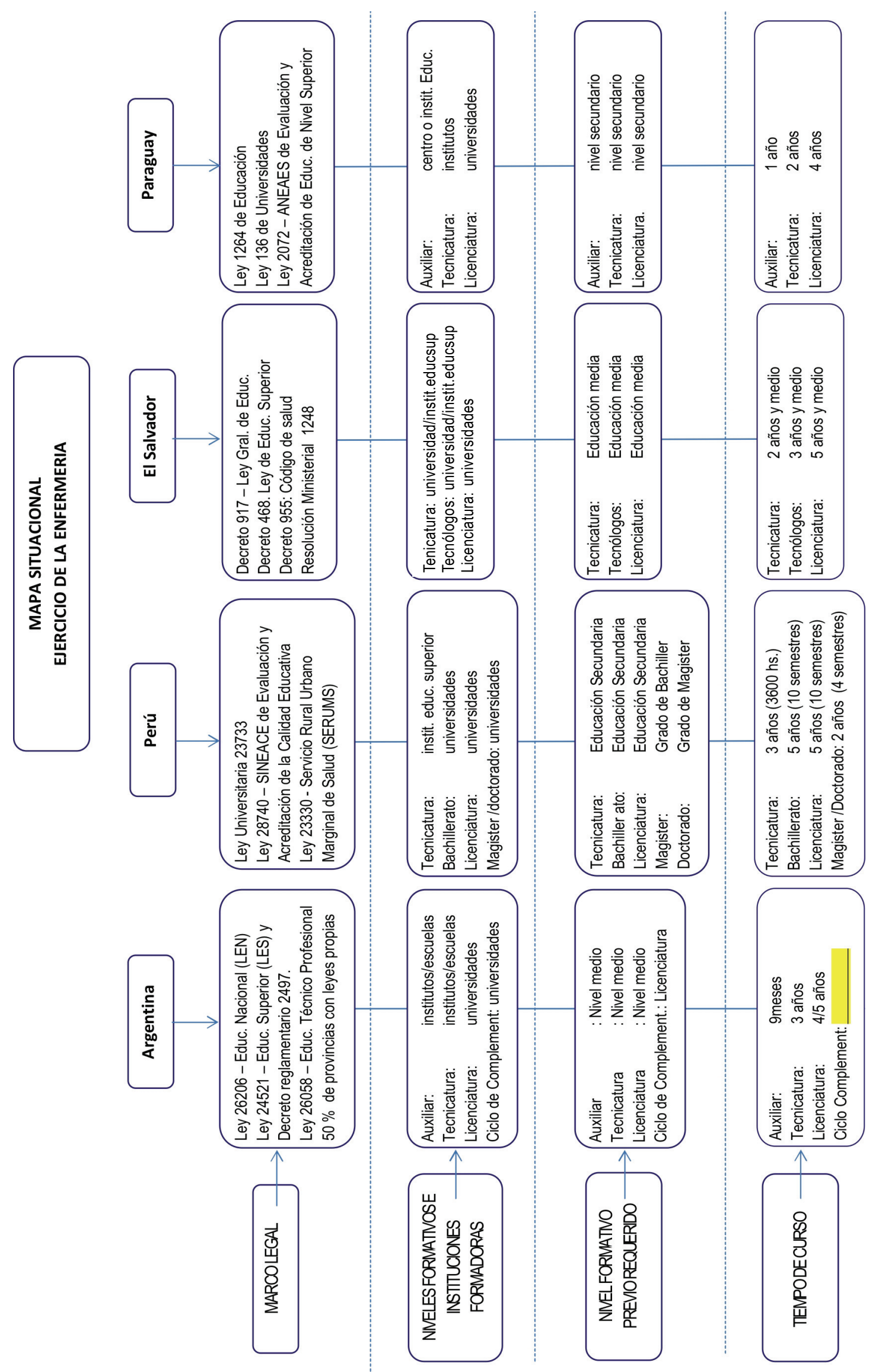




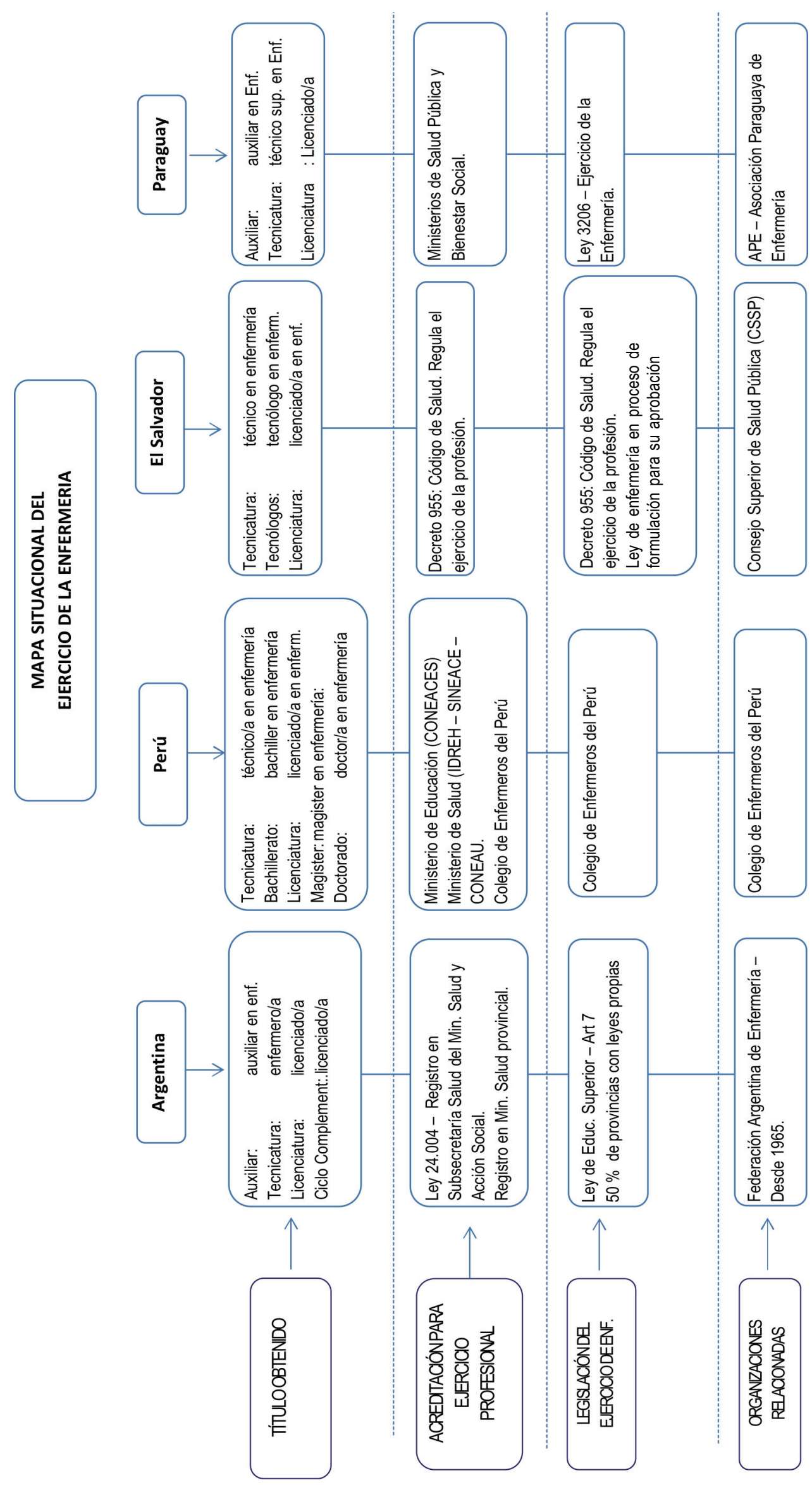


puntos de vista de la Unión Europea».

\section{REFERENCIAS BIBLIOGRÁFICAS}

1. Organización Internacional del Trabajo. Convenio No. 149 sobre Personal de Enfermería. 1977. [Citado 10 de julio de 2012]. Disponible en: http://webfusion. ilo.org/public/db/standards/normes/appl/appldisplayConv.cfm?conv $=\mathrm{C} 149$ \& hdroff $=1$ \&lang $=\mathrm{ES}$

2. Ley 24.004. Régimen legal del ejercicio de la enfermería. BUENOS AIRES, 26 de septiembre de 1991. Boletín Oficial, 28 de octubre de 1991. Vigentes. Decreto Reglamentario. Decreto Nacional 2.497/93.

3. Colegio de Enfermeros del Perú. Estatuto y Reglamento, Ley del trabajo de la enfermera, Código de Ética y Deontología. 2008. Disponible en: http:// www.cep.org.pe/beta/download/estatuto2009.pdf

4. Gobierno del Perú. Código Penal. Ministerio de Justicia. Disponible en: http://spij.minjus.gob.pe/CLP/ contenidos.dll? $\mathrm{f}=$ templates $\&$ fn=default codpenal.htm\&vid=Ciclope:CLPdemo

5. Constitución de la República del Salvador: Disponible en: http://www.constitution.org/cons/elsalvad.htm

6. República de El Salvador. Decreto 2699. Disponible en: http://www.seguridad.gob.sv/observatorio/ legislacion/LEY\%20DEL\%20CONSEJO\%20S UPERIOR\%20DE\%20SALUD\%20PUBLICA\%2 0Y\%20DE\%20LAS\%20JUNTAS\%20DE\%20VIGILAN CIA\%20DE\%20LAS\%20PROFESIONES\%20MEDICA.htm

7. Asamblea Legislativa de la República de El Salvador. Decreto 468. Ley de Educación Superior.

8. República de El salvador. Decreto 955: Código de salud. DL-DEC.e573. Diario Oficial (1988).

9. Congreso de la Nación Paraguaya. Ley No 3.206. Del ejercicio de la Enfermería. Poder Legislativo (2007).

10. Ministerio de Salud Pública y Bienestar Social. Reglamentación de la Ley 3.206 Del ejercicio de la Enfermería. Decreto 11.381/2007.

11. Organización de las Naciones Unidad para la Educación, Ciencia y la Cultura - UNESCO, editor. 50 years for education [Internet]. Paris: UNESCO; [citado 01 de septiembre de 2012]. Disponible en: http:/ /unesdoc.unesco.org/images/0011/001102/ 110264eb.pdf
12. Organización de las Naciones Unidad para la Educación, Ciencia y la Cultura - UNESCO. [Internet]. París: La Organización; c1995-2012 [citado 01 de septiembre de 2012]. La Educación Superior; [aprox. 1 p.]. Disponible en: http:// www.unesco.org/new/es/education/themes/ strengthening-education-systems/higher-education/

13. Congreso de la Nación - República de Argentina. Ley 26.206. Ley de Educación Nacional. El Senado y Cámara de Diputados de la Nación Argentina (2006).

14. Congreso de la Nación - República de Argentina. Ley 24.521. Ley de Educación Superior. El Senado y Cámara de Diputados de la Nación Argentina (1995).

15. Congreso de la Nación - República de Argentina. Ley 26.058. Ley de Educación Superior. El Senado y Cámara de Diputados de la Nación Argentina (2005).

16. Congreso de la República del Perú. Constitución Política del Perú. Lima: Oficialía Mayor. 1993.

17. Congreso de la República del Perú. Ley 23733. Ley Universitaria. Diario Oficial «El Peruano» (1983).

18. Congreso de la República del Perú. Ley 28740. Ley «Sistema Nacional de Evaluación, Acreditación y Certificación de la Calidad Educativa-SINEACE». Diario Oficial «El Peruano» (1983).

19. Asamblea Legislativa de la República de El Salvador. Decreto No 917. Ley General de Educación. (1990).

20. Agencia Nacional de Evaluación y Acreditación de la Educación Superior [Internet]. Asunción: ANAES. Disponible en: http://www.aneaes.gov.py/moodle/

21. Ley Nro. 1264. General de Educación del 21 de abril de 1998. Ley Nro. 136 de Universidades del mes de marzo de 1993.

\section{Correspondencia}

Yesenia Musayón Oblitas

Jr. Miguel Baquero 251 - Lima-Perú

Correo electrónico: flor.musayon@upch.pe

Forma de citar este artículo: Musayón-Oblitas Y, Farro-Peña G, Torres-Deza C, Alayo-Sarmiento M, Loncharich-Vera N, Zambonini S, Felippa G, Spadafora S, Osa M, Robles N, Roldan V, Figueroa-Gallardo T, Godines-Valencia C. Análisis comparativo de las leyes para el ejercicio de la Enfermería en Paraguay, El Salvador, Argentina y Perú. Rev enferm Herediana. 2012;5(2):133142. 